\title{
The Application of CAD/CAM in the Design of Industrial Products
}

\author{
Hequn Liu \\ Xianning Vocational Technical College, Hubei 437100, China
}

Keywords: CAD/CAM; industrial products; design; application

\begin{abstract}
CAD/CAM aided all kinds of design with computer aid, combining traditional design and manufacture, and perfected a highly unified information processing mode. With the rapid development of CAD/CAM technology, the process of product design and manufacture is more perfect, and the cost of design is reduced, which plays a very important role in industrial product design. This paper mainly analyzes the application of CAD/CAM in industrial products, so as to improve the design effect.
\end{abstract}

The design of industrial products is produced in the production of industrial products based on the model, in the production of modern industrial products, whether in design or in the mass production of products, is a very complex process, it must rely on a lot of technical talents to complete the task, and the design modification of the link is complex. Traditional design technology cannot meet the needs of modern design. The rational application of CAD/CAM technology can make up for the shortcomings of the traditional design, and unify the social and economic benefits [1].

\section{The concept of CAD/CAM}

CAD is a computer aided design software, using the computer software and hardware combination for product design or engineering, this technology combines computer features, the design method has been optimized, not only will combine the characteristics of human and computer, can make the design experience of people fully applied in the judgment the design concept, effective control of the design process. CAD plays a very important role in the analysis of the product, the design of the process and the digital control. On the basis of CAD application, CAM, as a computer aided manufacturing software, is an extension technology of product manufacturing. With the help of computer technology, the manufacturing process of products is monitored, so the integration process of product manufacturing is analyzed, and the path of cutter is simulated.

On the basis of the application of computer aided software, these two technologies should be closely coordinated so that they can restrict and coordinate each other.

\section{The development of technology in modern industry}

With the rapid development of modern industry, the application of CAD/CAM technology is also developing towards standardization. It has been applied in machinery manufacturing, electronics and chemical industry. The analysis of link in the development trend of CAD/CAM, which mainly realizes the characteristics of integration, based on the information technology and the rapid development of automation technology, the integration of manufacturing technology in two, the formation of a systematic scale, has been applied in mass production. With the continuous application of CAD/CAM technology, it will develop in the direction of intelligence in the future, and can improve all kinds of creative activities in the design. Now, the intelligent development of CAD/CAM should be combined with information technology to accurately analyze the intention of the designers and to automatically detect the unreasonable places in the design. 


\section{CAD/CAM Application in modern industry}

With the rapid development of CAD/CAM technology, it has been widely applied in mold manufacturing. It can enhance the intensive reading of dies, reduce the number of trial molds effectively, reduce the design cycle, and improve the efficiency of die design steadily.

In the design process, mainly adopts the serial design method, this design method is widely used in mold design, mold concurrent design, mold manufacturing quality can be improved, for each product development cycle of the mould function perfectly. In the mold design, the design is divided into the analysis of the functional requirements of the mould, the design of the plastic parts, the manufacture of the mould and so on. Each stage is inseparable. If a stage is not smooth, the cost of the whole process will be improved, and the quality of the mold can't be guaranteed. In every link of the mold design, the quality of the injection parts can not be improved if the error of the mold is relatively large [2].

In the process of molding part design, we can use CAD/CAM software and 3D modeling method to make the mold design link more three-dimensional. On the basis of the analysis of the demand of the product, the type of gate, the type of mold frame and the way of core pulling are analyzed. After the improvement of the basic design of the mold design, the CAM software plays a role in this way, which mainly plays the role of flow, cooling and pressure keeping, so that the cooling system and pouring system are more perfect. If the result of CAE analysis can't meet the requirements of production, it can also change the production plan in the production process, and make the injection process more perfect through multiple simulations.

In the CAM analysis, the scientific evaluation of the design, in the aspects of the design of the die structure, the cooling system and pouring system size requirements, design demoulding mechanism, with the design of insert and slide block, at this point, the application of CAD software. In the application of CAD software, the simulation NC machining is mainly carried out, and then the test production is carried out after the analysis of the process parameters according to the actual assembly [3].

Modeling of injection molded parts

The modeling of injection molded parts is completed on CAD software. Through the establishment of CAD platform, the structure and characteristics of injection molded parts are analyzed, and the size of precise injection parts is analyzed. We also need to collect a large number of material attributes, physical properties and process information. In the modeling process, we basically choose the type of plastic, analyze the performance of plastic parts, and combine the requirements of product shape and structural design, so as to improve the injection molding process. In the modeling part of injection molding, we should not only meet the needs of users, but also improve the processing of plastic products in subsequent design. After the plastic part model is completed, the mold is designed with CAD software to realize the cavity of the mold.

Generally, plastic parts modeling is divided into two different situations. First, modeling the original plastic parts. Based on the analysis of the structure and size of the plastic parts, combined with the size and structure, the model is established. Secondly, in view of the customer's demand, the plastic parts are established and the specific parameters are designed. In the link of the application of the compound modeling technology, it provides a reliable basis for the design of the injection product in view of the characteristics of the modeling. Today, these two modeling methods provide a guarantee for modeling, and people can not only collect product information, but also collect mold information.

In the design of CAD application, the plastic parts can show the three-dimensional model in the design, the overall design scheme, to determine a good mold, determine the number of good cavity, layout of cavity position, and then through the pouring system, determine pouring way, analysis of whether to adopt a separation in pouring in, whether to use a knife to the side core pulling mechanism. In the design of the cooling system, the type and specifications of the mold frame are determined. In the design of precision industrial products, the requirement for forming is very high. In the design link, it is necessary to ensure that the cooling system can be poured and cooled evenly. 
Secondly, in the links of forming part design, there are several key directions in the design of the forming part, and the quality and dimension accuracy of the formed surface should be controlled, otherwise, it will have a great influence on the surface quality of the product. In the process of precision casting, the size of the formed parts is as small as possible. A unified injection mold can not be used, and the size accuracy should be controlled reasonably. In the analysis of the size of the drawing surface of the plastic parts and the size of the drawing surface of the mold, the actual size of the two should be obtained. On the basis of the manufacture, the products of the injection molding should be obtained. After the analysis of the actual size of the product, make sure that the size is within the reasonable range of tolerance. This theory has a good effect on the control of die wear, manufacturing error and shrinkage. Thirdly, in the links of structural parts design, we must choose the standard parts of mould bases, and analyze the stiffness and strength of mould based on the amount of injection pressure, so as to prevent the two changes, and lead to the accuracy of injection moulds. Although die structure parts do not directly participate in plastic forming, it has an effect on the precision of die cavity [4].

In the link of injection mold CAE application, quantitative analysis is made for every stage of plastic forming, and the error of mould is corrected in time. According to the heat transfer and rheology of plastics, the numerical value is calculated by computer, so that it can be directly visualized by graphics, and the local temperature field, pressure field and cooling process are analyzed. With the help of CAE technology, the die is designed, the defects of the forming are predicted and the results of warpage are predicted.

In the links of traditional CAE injection software, the flow of central part of the plastic part is simulated. This technology combines the abstract analysis of thin-walled plastic parts, because the thin wall is a combination of straight surface and curved surface, so it is also called the neutral surface. A three-dimensional mesh is established on the neutral surface, and the finite element analysis is carried out. The numerical value of the pressure field and temperature field is accurately analyzed, and different colors are marked on the center layer. This technology is well applied to complex plastic parts, but there is also a problem. Because of the complex structure of three-dimensional plastic parts, the operation is more complex and the result of the analysis is very poor.

The 3D simulation of the link, its application is very extensive, this technique does not require the use of the center layer, the 3D model directly on the analysis of the technology in the application process, using two-dimensional finite element method, the three dimensional solid plastic analysis. Metro will a two-dimensional map in solid surface. If the thickness of the plastic part is smaller, then the symmetric information can be formed after the triangular mesh is paired. The thickness information is calculated by the finite element method, and the different colors can be displayed by the three-dimensional entity [5].

The 3D model of flow play a very large role, but there are also problems in the two-dimensional finite element analysis in the link, if not on the liquid plastic thickness and direction, can not analyze the performance in the micro molding process, three-dimensional flow simulation with 3D solid finite element, will produce the calculation results can be directly displayed in the 3D entity.

\section{The foundation of mould processing for industrial products}

In the process of industrial parts processing, after analyzing the size and characteristics of parts and designing technological parameters, we can form digital codes. When these codes are directly input into machine tools, we can control the operation of machine tools.

In the general CNC machine tools, the characteristics of NC machining are mainly of high precision and stable processing quality. The way of NC machining is very efficient, and it will not bring too high requirement to the operators of machine tools. The machining accuracy of industrial products can be guaranteed, and the consistency of parts processing can be guaranteed. In the processing link, it is very convenient to manage and prevent the fixture from taking too much time. In the design, it is very convenient to realize the concept of flexible machining [6].

With the continuous improvement of industrial products, its performance is developing in a 
diversified way. When customers are in mold manufacturing, they need to improve mold production in a short time. Now, the electronic technology has achieved the highly effective development. The numerical control technology can be guaranteed in the development of the hardware numerical control technology, and the computer is used to control the processing flow, so that the application scope of the numerical control technology is more extensive. With the continuous improvement and development of NC machining technology, with the help of CAD/CAM system, the manufacturing cycle is reduced in the processing of industrial products.

\section{Theoretical basis of NC machining}

In the work of numerical control programming, the trajectory of the props should be formed, and the trajectory should be presented in the form of discrete points. The path of NC tool can be represented by points, lines and planes. In the actual industrial product processing, entity processing is relative to contour processing. In general, the way of slice cutting is used in entity processing, which improves the way of processing horizontal plane. In the process of NC tool processing under the characteristic background, operators do not need to do practical operation on points, lines and planes, so as to program for features, so that the efficiency of programming is greatly improved. There are all kinds of parameters in the feature, but the time of tool path used in the characteristic background is not long.

In the processing of the entity of industrial products, the main method is to use the feature processing, but there is still a difference between the solid processing and the feature processing. The feature processing is to reinforce the parts of the industrial products, and the way of operation is familiar to people. However, in the process of entity processing, with the help of geometric problems, functional semantic information will not be presented. In the actual processing of industrial products, it is often to use a variety of processing methods, on the basis of turning processing, but also to milling the disease palace. The machining of the parts is generally refined to the parts, and the processing precision is required by the feature processing.

In the process of processing industrial products, a number of numerical control instructions are required. In order to ensure the accuracy of program operation and prevent the collision of industrial products in processing links, we should have advanced tests in specific production links. This method is a waste of raw materials, resulting in higher production costs. In the display of track based method application, using a color simulation processing, the cutting trajectory was simulated, using wood, paraffin and other materials to replace 3D and multidimensional processing way, this way also has some problems, will lead to the use of a large number of CNC lathe. Therefore, the computer should be used to simulate the cutting way through the graphic display.

\section{Conclusions}

This technology has been widely used in the processing of industrial products. The two parties cooperate with each other to improve the processing efficiency of industrial products.

\section{References}

[1] Barrow L. Digital Design Pedagogy - Basic Design - CADCAM Space Box Exploration[J]. SIGraDi 2006 - [Proceedings of the 10th Iberoamerican Congress of Digital Graphics] Santiago de Chile - Chile 21-23 November 2006, pp. 127-130, 2006.

[2] Risdiyono. The Applications of Cad/Cam Technology for Small Industries in Developing Creative Culture Design of Art Jewelery [J]. 2008.

[3] Zhu L. On the Application of CAD/CAM Software in Mold Design and Manufacture[J]. Modern Manufacturing Technology \& Equipment, 2007.

[4] Cai N. Study on the Industrial Application of CAD/CAM Aided Design[J]. Journal of Chongqing University of Science \& Technology, 2013. 
[5] Gaunt G. The application of CAD/CAM to design and manufacture of winged sails[C]// Institution of Engineers, Australia, 1987.

The first author: Hequn Liu Male, Hunan province, November May, 1962. Bachelor's degree of the university, Associate professor, Mechanical design and manufacturing. 